\title{
Comparison of Adomian Decomposition and Taylor Expansion Methods for the Solutions of Fractional Integro-Differential Equations
}

\author{
M. H. Saleh \\ Mathematics Department \\ Faculty of Science \\ Zagazig University, Egypt
}

\author{
S. M. Amer \\ Mathematics Department \\ Faculty of Science \\ Zagazig University, Egypt
}

\author{
M. A. Shaalan \\ Basic Science Department \\ Higher Technological Institute \\ Tenth of Ramadan City, Egypt
}

\begin{abstract}
In this paper will be compared between Adomian decomposition method (ADM) and Taylor expansion method (TEM) for solving (approximately) a class of fractional integro-differential equations. Numerical examples are presented to illustrate the efficiency and accuracy of the proposed methods.
\end{abstract}

\section{General Terms}

Numerical solutions, Fractional integro-differential equations.

\section{Keywords}

Fractional integro-differential equations, Adomian decomposition method, Taylor expansion method, Caputo fractional derivative, Riemann-Liouville.

\section{INTRODUCTION}

In this paper will be taken the fractional integro-differential equations with a Caputo fractional derivative of the type

$$
\begin{aligned}
& D_{*}^{q} y(t)=P(t) y(t)+f(t)+\int_{0}^{t} K(t, x) y(x) d x, t \\
& \in[0,1]
\end{aligned}
$$

with initial condition

$$
y(0)=\alpha, \quad n-1<q \leq n, n \in N
$$

where $D_{*}^{q}$ is Caputo's fractional derivative and $\alpha$ is a parameter describing the order of the fractional derivative. Such kind of equations arise in the mathematical modelling of various physical phenomena, such as heat conduction in materials with memory. Moreover, these equations are encountered in combined conduction, convection and radiation problems $[6,11,16]$. So far, fractional calculus as well as fractional differential equations have received increasing attention in recent years. The existence and uniqueness of solutions to fractional differential equations have been investigated [2,7,9,14]. In addition, when $\alpha \in N$, $\mathrm{Eq}(1.1)$ reduces to linear integro-differential equation and the numerical methods for this equation have been extensively studied by many authors $[8,12,15]$. There are many methods for seeking approximate solutions such as collocation method, the fractional differential transform method, Legendre wavelet method, Taylor expansion method and Adomian decomposition method, See $([1,4,5,10,13])$. The outline of this paper is as follows: In section 2, we present some definitions.
Section 3, contains the application of the Adomian decomposition method. Section 4, contains the application of the Taylor expansion method. Finally, Sec.5 devoted to illustrate some numerical examples on mentioned methods.

\section{SOME DEFINITIONS AND NOTATIONS}

Definition 2.1. A real function $f(x), x>0$, is said to be in the space $C_{\alpha}, \alpha \in R$, if there exists a real number $p>\alpha$, such that $f(x)=x^{p} f_{1}(x)$, where $f_{1}(x) \in C[0, \infty)$.

Definition 2.2. A real function $f(x), x>0$, is said to be in the space $C_{\alpha}^{k}, k \in N \cup\{0\}$, if $f^{k} \in C_{\alpha}$.

Definition 2.3. [7] $D^{q}$ (q is real) denotes the fractional differential operator of order $\mathrm{q}$ in the sense of RiemannLiouville, defined by

$$
\begin{aligned}
& D^{q} f(t) \\
& =\left\{\begin{array}{l}
\frac{1}{\Gamma(n-q)} \frac{d^{n}}{d t^{n}} \int_{0}^{t} \frac{f(x)}{(t-x)^{q-n+1}} d x, 0 \leq n-1<q \leq n \\
\frac{d^{n} f(t)}{d t^{n}}, q=n \in N .
\end{array}\right.
\end{aligned}
$$

Definition 2.4. [7] $I^{q}$ denotes the fractional integral operator of order $\mathrm{q}$ in the sense of Riemann-Liouville, defined by

$$
\begin{aligned}
& I^{q} f(t)=D^{-q} f(t) \\
& \left\{\begin{array}{l}
\frac{1}{\Gamma(q)} \int_{0}^{t} \frac{f(x)}{(t-x)^{1-q}} d x, q>0, \\
f(t), q=0 .
\end{array}\right.
\end{aligned}
$$

Definition 2.5. [7] Let $f \in C_{-1}^{n}, n \in N$. Then the Caputo fractional derivative of $f(t)$, defined by

$$
\begin{aligned}
D_{*}^{q} f(t) & \left\{\begin{array}{l}
\frac{1}{\Gamma(n-q)} \int_{0}^{t} \frac{f^{(n)}(x)}{(t-x)^{q-n+1}} d x, 0 \leq n-1<q \leq n, \\
\frac{d^{n} f(t)}{d t^{n}}, q=n \in N .
\end{array}\right.
\end{aligned}
$$


Now will be introduced some basic properties of fractional operator are listed below [7]:for $f \in C_{\alpha}, \alpha \geq-1, \mu \geq 1, \eta \geq$ $0, \beta>-1, \delta \geq 0$ :

(I) $I^{\mu} \in C_{0}$.

(II) $I^{\eta} I^{\delta} f(x)=I^{\eta+\delta} f(x)=I^{\delta} I^{\eta} f(x)$.

(III) $D^{\eta} D^{\delta} f(x)=D^{\eta+\delta} f(x)$.

$(\mathrm{IV}) D^{\delta} I^{\delta} f(x)=f(x)$

$(\mathrm{V}) I^{\delta} D_{*}^{\delta} f(x)=f(x)-\sum_{k=0}^{n-1} f^{(k)}\left(0^{+}\right) \frac{t^{k}}{k !}, 0 \leq n-1<\delta \leq$ $n \in N$.

$(\mathrm{VI}) I^{\delta} x^{\beta}=\frac{\Gamma(\beta+1)}{\Gamma(\beta+1+\delta)} x^{\beta+\delta} ; x>0$.

$(\mathrm{VII}) D^{\delta} x^{\beta}=\frac{\Gamma(\beta+1)}{\Gamma(\beta+1-\delta)} x^{\beta-\delta} ; x>0$.

\section{ADOMIAN DECOMPOSITION}

\section{METHOD}

Consider the equation (1.1) with initial condition (1.2) where $D_{*}$ is the operator defined in (2.3).Operating with $I^{q}$ on both sides of the equation (1.1) as follows:

$$
\begin{gathered}
y(t)=\sum_{k=0}^{n-1} y^{(k)}\left(0^{+}\right) \frac{t^{k}}{k !}+I^{q}[P(t) y(t)+f(t)+ \\
\left.\int_{0}^{t} K(t, x) y(x) d x\right] .
\end{gathered}
$$

Adomain decomposition method defines the solution by the series:

$y(t)=\sum_{n=0}^{\infty} y_{n}(t)$

where the components $y_{0}, y_{1}, y_{2}, \ldots \ldots$ are determined recursively by

$y_{0}(t)=\sum_{k=0}^{n-1} y^{(k)}\left(0^{+}\right) \frac{t^{k}}{k !}+I^{q} f(t)$,

$y_{k+1}(t)=I^{\alpha}\left[P(t) y_{k}(t)\right]+I^{q}\left[\int_{0}^{t} K(t, x) y_{k}(x) d x\right]$.

Decomposition method suggests that $0^{\text {th }}$ component $y_{0}(t)$ be defined by the initial conditions and the function $f(t)$ as described above. The other components namely $y_{1}, y_{2}, \ldots$ etc. are derived recurrently.

\section{TAYLOR EXPANSION METHOD}

Consider the equation (1.1) with initial condition (1.2). First, integrate both sides of the equation (1.1) with respect to $t$ for $n$ times. Using Def. (2.4) as follows:

$$
\begin{aligned}
& \int_{0}^{t} \frac{(t-x)^{n-q-1}}{\Gamma(n-q)} y(x) d x=\int_{0}^{t} \frac{(t-x)^{n-1} P(x)}{(n-1) !} y(x) d x+ \\
& \int_{0}^{t} \frac{(t-x)^{n-1} f(x)}{(n-1) !} d x+\frac{1}{(n-1) !} \int_{0}^{t} y(x) \int_{x}^{t} K(s, x)(s- \\
& x)^{n-1} d s d x+Q_{n}(t) .
\end{aligned}
$$

Next, Assume that $y \in C^{m+1}[0,1], y(x)$ can be represented as Taylor expansion $m^{\text {th }}$ order as follows:

$$
\begin{gathered}
y(x)=y(t)+y^{\prime}(t)+\ldots \ldots+y^{(m)}(t) \frac{(x-t)^{m}}{m !}+ \\
\frac{y^{(m+1)}(\eta(x))}{(m+1) !}(x-t)^{m+1} .
\end{gathered}
$$

Where $t<\eta(x)<x$. It is readily shown that the Lagrange reminder $\frac{y^{(m+1)}(\eta(x))}{(m+1) !}(x-t)^{m+1}$ is sufficiently small for large enough $\mathrm{m}$ provided that $y^{(m+1)}(x)$ is uniformly bounded function for any $\mathrm{m}$ on the interval $[0,1]$. So, we will neglect the remainder and the truncated Taylor expansion $\mathrm{y}(\mathrm{x})$ as follows:

$y(x) \approx \sum_{j=0}^{m} y^{(j)}(t) \frac{(x-t)^{j}}{j !}$.

Substituting the approximate expression (4.3) for $\mathrm{y}(\mathrm{x})$ into equation (4.1),

$\sum_{j=0}^{m} \int_{0}^{t} \frac{(t-x)^{n-q-1}}{\Gamma(n-q)} y^{(j)}(t) \frac{(x-t)^{j}}{j !} d x=$

$\sum_{j=0}^{m} \int_{0}^{t} \frac{(t-x)^{n-1} P(x)}{(n-1) !} y^{(j)}(t) \frac{(x-t)^{j}}{j !} d x+\int_{0}^{t} \frac{(t-x)^{n-1} f(x)}{(n-1) !} d x+$

$\sum_{j=0}^{m} \frac{1}{(n-1) !} \int_{0}^{t} y^{(j)}(t) \frac{(x-t)^{j}}{j !} \int_{x}^{t} K(s, x)(s-x)^{n-1} d s d x+$

$Q_{n}(t)$.

Or further;

$k_{00}(t) y(t)+k_{01}(t) y^{\prime}(t)+\ldots \ldots \ldots+k_{0 m}(t) y^{(m)}(t)$

$=f_{(n)}(t)$,

where

$k_{0 j}=\frac{(-1)^{j} t^{n+j-q}}{(n+j-q) \Gamma(n-q) j !}-\frac{1}{(n-1) ! j !} \int_{0}^{t}(x-t)^{j} \int_{x}^{t} K(s, x)(s-$

$x)^{n-1} d s d x-\frac{(-1)^{j}}{(n-1) !} \int_{0}^{t} P(x)(t-x)^{n+j-1} d x, j=$

$0,1, \ldots, m$,

$f_{(n)}(t)=\frac{1}{(n-1) !} \int_{0}^{t}(t-x)^{n-1} f(x)+Q_{n}(t)$.

Thus equation (4.1) becomes an $m^{\text {th }}$ order, linear, ordinary differential equation with variable coefficients for $y(t)$ and it 's derivative up to $\mathrm{m}$. So, will be determined $y(t), \ldots, y^{(m)}(t)$ by solving linear equations. Now, other $m$ independent linear equations for $y(t), \ldots, y^{(m)}(t)$ are needed. This can be achieved by integrating both sides of the equation (4.1) with respect to $\mathrm{t}$ from 0 to $\mathrm{s}$ and changing the order of the integration.

$$
\begin{aligned}
& \int_{0}^{t} \frac{(t-x)^{n-q}}{\Gamma(n+1-q)} y(x) d x= \\
& \int_{0}^{t} \frac{(t-x)^{n} P(x)}{(n) !} y(x) d x+\int_{0}^{t}\left(\frac{(t-x)^{n} f(x)}{(n) !}+Q_{n}(x)\right) d x+ \\
& \frac{1}{(n) !} \int_{0}^{t} y(x) \int_{x}^{t} K(s, x)(s-x)^{n} d s d x .
\end{aligned}
$$


Where replace variable s with t. Applying Taylor expansion again and substituting (4.3) for $\mathrm{y}(\mathrm{x})$ into equation (4.8) gives

$k_{10}(t) y(t)+k_{11}(t) y^{\prime}(t)+\ldots \ldots \ldots+k_{1 m}(t) y^{(m)}(t)=$

$f_{(n+1)}(t)$,

where

$k_{1 j}=\frac{(-1)^{j} t^{n+j+1-q}}{(n+j+1-q) \Gamma(n+1-q) j !}-\frac{1}{n ! j !} \int_{0}^{t}(x-t)^{j} \int_{x}^{t} K(s, x)(s-$ $x)^{n} d s d x-\frac{(-1)^{j}}{n ! j !} \int_{0}^{t} P(x)(t-x)^{n+j} d x, j=$

$0,1, \ldots, m$,

$f_{(n+1)}(t)=\int_{0}^{t}\left(\frac{(t-x)^{n} f(x)}{n !}+Q_{n}(x)\right) d x$.

By repeating the above integration process for i $\left(i \in N^{+}, 1<\right.$ $i \leq m)$ times.

$k_{i 0}(t) y(t)+k_{i 1}(t) y^{\prime}(t)+\ldots \ldots \ldots+k_{i m}(t) y^{(m)}(t)=$

$f_{(n+i)}(t)$,

where

$k_{i j}=$

$\frac{(-1)^{j} t^{n+j+i-q}}{(n+j+i-q) \Gamma(n+i-q) j !}-\frac{1}{(n+i-1) ! j !} \int_{0}^{t}(x-t)^{j} \int_{x}^{t} K(s, x)(s-$

$x)^{n+i-1} d s d x-\frac{(-1)^{j}}{(n+i-1) ! j !} \int_{0}^{t} P(x)(t-x)^{n+j+i-1} d x$,

$f_{(r)}(t)=\int_{0}^{t} f_{(r-1)}(x) d x, r>n+1, r \in N^{+}$.

Therefore, equations (4.6), (4.9), (4.12) form a system $(m+1)$ linear equations for $(\mathrm{m}+1)$ unknown functions $y(t), \ldots, y^{(m)}(t)$. For simplicity, the system can be written as:

$K_{m m}(t) Y_{m}(t)=F_{m}(t)$.

Where $K_{m m}(t)$ is an $(m+1) \times(m+1)$ square matrix function in $\mathrm{t}, Y_{m}(t), F_{m}(t)$ are two vectors of length $(\mathrm{m}+1)$, and these are defined as

$$
K_{m m}(t)=\left(\begin{array}{llll}
K_{00}(t) & K_{01}(t) & \ldots & K_{0 m}(t) \\
K_{10}(t) & K_{11}(t) & \ldots & K_{1 m}(t) \\
\vdots & \vdots & \ddots & \vdots \\
K_{m 0}(t) & K_{m 1}(t) & \ldots & K_{m m}(t)
\end{array}\right),
$$

$$
Y_{m}(t)=\left(\begin{array}{l}
y(t) \\
y^{\prime}(t) \\
\vdots \\
y^{(m)}(t)
\end{array}\right), F_{m}(t)=\left(\begin{array}{c}
f_{(n)}(t) \\
f_{(n+1)}(t) \\
\vdots \\
f_{(n+m)}(t)
\end{array}\right) .
$$

By using Cramer's rule, obtain the approximate solution y(t) as:

$$
y(t)=\frac{\operatorname{det}\left(M_{m m}(t)\right)}{\operatorname{det}\left(K_{m m}(t)\right)} .
$$

Where

$$
M_{m m}(t)=\left(\begin{array}{llll}
f_{n}(t) & K_{01}(t) & \ldots & K_{0 m}(t) \\
f_{n+1}(t) & K_{11}(t) & \ldots & K_{1 m}(t) \\
\vdots & \vdots & \ddots & \vdots \\
f_{n+m}(t) & K_{m 1}(t) & \ldots & K_{m m}(t)
\end{array}\right) .
$$

\section{NUMERICAL EXAMPLES}

In this paper, according to Adomian decomposition method, choose $\mathrm{m}=3$.So, calculate the numerical results $y_{\text {App.(t) }}$ by using equations (3.3), (3.4) and the absolute errors can be written as $e=\left|y_{\text {App. }}(t)-y_{\text {exact }}(t)\right|$, where $y_{\text {App. }}(t)=$ $y_{0}(t)+y_{1}(t)+y_{2}(t)$. While, according to Taylor's method, choose $\mathrm{m}=3$. So, calculate the numerical results $y_{A p p}(t)$ by using equations (4.18) and the absolute errors can be written as $e=\left|y_{\text {App. }}(t)-y_{\text {exact }}(t)\right|$. All results are obtained by using Maple 16.

Example 5.1. Consider the fractional integro-differential equation:

$$
\begin{gathered}
D^{0.5} y(t)=\frac{t^{0.5}}{\Gamma(1.5)}+\frac{1}{2} t e^{t}\left(\sin (t)-\cos (t)+\frac{\sin (t)}{t}\right)- \\
e^{t} \sin (t) y(t)+\int_{0}^{t} e^{s} \cos (\mathrm{s}) y(s) d s, \\
y(0)=0 .
\end{gathered}
$$

With the exact solution $y(t)=t$. (Show table 1, and figure 1.) 
Table 1. The absolute errors of Example 5.1.

\begin{tabular}{|l|l|l|}
\hline$t$ & Abs.E (ADM) & Abs.E (TEM) \\
\hline 0.1 & $2.108 \mathrm{E}-7$ & $1.0 \mathrm{E}-10$ \\
\hline 0.2 & $1.15952 \mathrm{E}-5$ & $1.12 \mathrm{E}-8$ \\
\hline 0.3 & $1.322509 \mathrm{E}-4$ & $3.0 \mathrm{E}-9$ \\
\hline 0.4 & $7.931674 \mathrm{E}-4$ & $2.7 \mathrm{E}-9$ \\
\hline 0.5 & $3.3390101 \mathrm{E}-3$ & $2.0 \mathrm{E}-9$ \\
\hline 0.6 & $1.12024541 \mathrm{E}-2$ & $2.67 \mathrm{E}-8$ \\
\hline 0.7 & $3.20227646 \mathrm{E}-2$ & $3.35 \mathrm{E}-8$ \\
\hline 0.8 & $8.11245207 \mathrm{E}-2$ & $3.38 \mathrm{E}-8$ \\
\hline 0.9 & $1.86775703 \mathrm{E}-1$ & $6.43 \mathrm{E}-8$ \\
\hline 1.0 & $3.97547118 \mathrm{E}-1$ & 0 \\
\hline
\end{tabular}

To avoid difficult fractional integral, take the truncated Taylor expansions for $\cos (t), \sin (t), e^{t}$ in the equation (5.1). But, by using modified decomposition method [3], and select $y_{0}(t)=$ $t$, obtain

$$
\begin{aligned}
y_{1}(t)=I^{0.5}\left(\frac{1}{2} t e^{t}\right. & \left.\sin (t)-\frac{1}{2} t e^{t} \cos (t)-\frac{1}{2} e^{t} \sin (t)\right) \\
& \left.+I^{0.5}\left(e^{t} \sin (t)\right) y_{0}(t)\right) \\
& +I^{0.5}\left(\int_{0}^{t} e^{s} \cos y_{0}(s) d s\right)=0
\end{aligned}
$$

$y_{n+2}(t)=0, n \geq 0$.

So that the solution in closed form $y(t)=t$.

Example 5.2. Consider the fractional integro-differential equation :

$$
\begin{gathered}
D^{0.75} y(t)=\frac{t^{0.25}}{\Gamma(1.25)}-t^{2}-\frac{t^{4}}{3}+t y(t)+\int_{0}^{t} t s y(s) d s, \\
y(0)=0 .
\end{gathered}
$$

With the exact solution $y(t)=t$. (Show table 2, and figure 2.)

Table 2. The absolute errors of Example 5.2.

\begin{tabular}{|c|c|c|}
\hline$t$ & Abs.E (ADM) & Abs.E (TEM) \\
\hline 0.1 & $2.019 \mathrm{E}-8$ & $1.0354 \mathrm{E}-7$ \\
\hline 0.2 & $1.5524 \mathrm{E}-6$ & $2.7244 \mathrm{E}-6$ \\
\hline 0.3 & $1.99559 \mathrm{E}-5$ & $1.81447 \mathrm{E}-5$ \\
\hline 0.4 & $1.237975 \mathrm{E}-4$ & $6.81375 \mathrm{E}-5$ \\
\hline 0.5 & $5.168022 \mathrm{E}-4$ & $1.844504 \mathrm{E}-4$ \\
\hline 0.6 & $1.6831889 \mathrm{E}-3$ & $3.991721 \mathrm{E}-4$ \\
\hline 0.7 & $4.6270796 \mathrm{E}-3$ & $7.196495 \mathrm{E}-4$ \\
\hline 0.8 & $1.12501689 \mathrm{E}-2$ & $1.0759817 \mathrm{E}-3$ \\
\hline 0.9 & $2.49296030 \mathrm{E}-2$ & $1.2179160 \mathrm{E}-3$ \\
\hline 1.0 & $5.13811204 \mathrm{E}-2$ & $5.203903 \mathrm{E}-4$ \\
\hline
\end{tabular}

But, by using modified decomposition method [3], and select $y_{0}(t)=t$, obtain

$$
\begin{aligned}
y_{1}(t)=I^{0.75}\left(-t^{2}-\right. & \left.\frac{t^{4}}{3}\right)+I^{0.75}\left(t y_{0}(t)\right) \\
& +I^{0.75}\left(\int_{0}^{t} t s y_{0}(s) d s\right)=0
\end{aligned}
$$

$y_{2}(t)=0$,

$y_{n+3}(t)=0, n \geq 0$.

So that the solution in closed form $y(t)=t$.

Example 5.3. Consider the fractional integro-differential equation :

$$
\begin{gathered}
D^{0.5} y(t)=(\cos (t)-\sin (t)) y(t)+f(t)+ \\
\int_{0}^{t} t \sin (s) y(s) d s \\
y(0)=0 . \\
f(t)=\frac{2 t^{1.5}}{\Gamma(2.5)}+\frac{t^{0.5}}{\Gamma(1.5)}+t(2-3 \cos (t)-t \sin (t)+ \\
\left.t^{2} \cos (t)\right)
\end{gathered}
$$

With the exact solution $y(t)=t^{2}+t$. (Show table 3, and figure 3.)

Table3. The absolute errors of Example 5.3.

\begin{tabular}{|c|c|c|}
\hline $\mathrm{t}$ & Abs.E (ADM) & Abs.E (TEM) \\
\hline 0.1 & $7.982452 \mathrm{E}-4$ & $5.626 \mathrm{E}-7$ \\
\hline 0.2 & $3.6471117 \mathrm{E}-3$ & $1.29901 \mathrm{E}-5$ \\
\hline 0.3 & $7.8054711 \mathrm{E}-3$ & $8.24971 \mathrm{E}-5$ \\
\hline 0.4 & $1.19599592 \mathrm{E}-2$ & $3.084884 \mathrm{E}-4$ \\
\hline 0.5 & $1.50115353 \mathrm{E}-2$ & $8.647433 \mathrm{E}-4$ \\
\hline 0.6 & $1.64762039 \mathrm{E}-2$ & $2.0151158 \mathrm{E}-3$ \\
\hline 0.7 & $1.6551653 \mathrm{E}-2$ & $4.123505 \mathrm{E}-3$ \\
\hline 0.8 & $1.5885982 \mathrm{E}-2$ & $7.650717 \mathrm{E}-3$ \\
\hline 0.9 & $1.5148298 \mathrm{E}-2$ & $1.3138091 \mathrm{E}-2$ \\
\hline 1.0 & $1.4554082 \mathrm{E}-2$ & $2.1177649 \mathrm{E}-2$ \\
\hline
\end{tabular}

To avoid difficult fractional integral take the truncated Taylor expansions for the trigonometric terms in terms in the equation (5.3) e.g. [ $\left.\cos t \approx 1-\frac{t^{2}}{2 !}+\frac{t^{4}}{4 !}, \sin t \approx t-\frac{t^{3}}{3 !}+\frac{t^{5}}{5 !}\right]$. But, by using a modified decomposition method [3], and select $y_{0}(t)=t^{2}+t$, obtain 


$$
\begin{array}{r}
y_{1}(t)=I^{0.5}(2 t-3 t \\
\left.+\cos (t)-t^{2} \sin (t)+t^{3} \cos (t)\right) \\
+I^{0.5}\left((\cos (t)-\sin (t)) y_{0}(t)\right) \\
+I^{0.5}\left(\int_{0}^{t} t \sin (s) y_{0}(s) d s\right)=0,
\end{array}
$$

$y_{n+2}(t)=0, n \geq 0$

So that the solution in closed form $y(t)=t^{2}+t$.

Example 5.4. Consider the fractional integro-differential equation :

$$
\begin{gathered}
D^{0.25} y(t)=\frac{6 t^{2.75}}{\Gamma(3.75)}-\frac{1}{5} t^{2} e^{t} y(t)+\int_{0}^{t} e^{t} s y(s) d s, \\
y(0)=0 .
\end{gathered}
$$

With the exact solution $y(t)=t^{3}$. (Show table 4. and figure 4.)

Table 4. The absolute errors of Example 5.4.

\begin{tabular}{|c|c|c|}
\hline $\mathrm{t}$ & Abs.E $(\mathrm{ADM})$ & Abs.E $(\mathrm{TEM})$ \\
\hline 0.1 & $\mathbf{0}$ & $7.2341 \mathrm{E}-9$ \\
\hline 0.2 & $\mathbf{0}$ & $5.97738 \mathrm{E}-7$ \\
\hline 0.3 & $\mathbf{0}$ & $7.97449 \mathrm{E}-6$ \\
\hline 0.4 & $\mathbf{0}$ & $5.173697 \mathrm{E}-5$ \\
\hline 0.5 & $\mathbf{0}$ & $2.224414 \mathrm{E}-4$ \\
\hline 0.6 & $\mathbf{0}$ & $7.350817 \mathrm{E}-4$ \\
\hline 0.7 & $\mathbf{0}$ & $2.0168252 \mathrm{E}-3$ \\
\hline 0.8 & $\mathbf{0}$ & $1.0226069379 \mathrm{E}-3$ \\
\hline 0.9 & $\mathbf{0}$ & $1.97594433 \mathrm{E}-2$ \\
\hline 1.0 & $\mathbf{0}$ & \\
\hline
\end{tabular}

According to Adomian decomposition method, find the exact solution because $y_{0}(t)=t^{3}$.

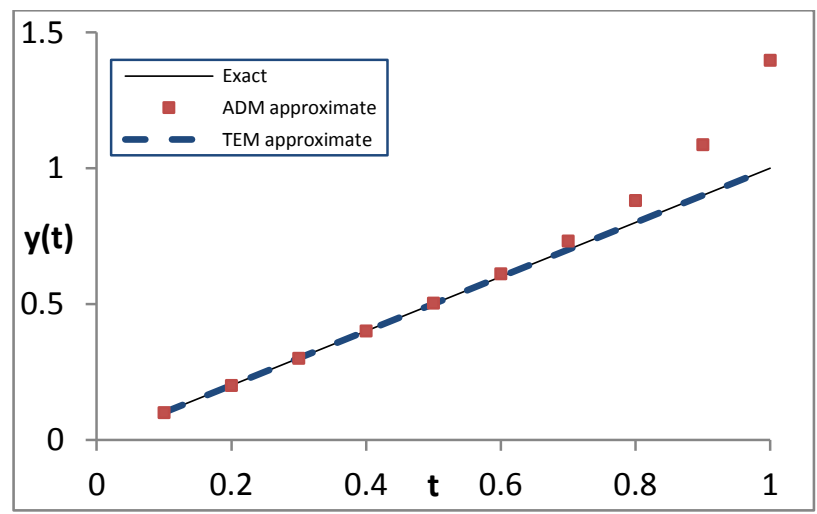

Fig.1. Comparison of approximate solutions obtained by ADM and TEM with exact solution of example 5.1.

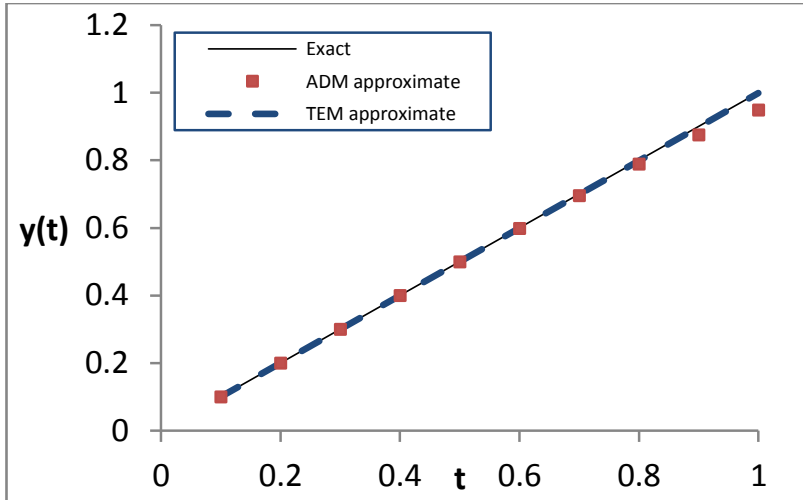

Fig.2. Comparison of approximate solutions obtained by ADM and TEM with exact solution of example 5.2.

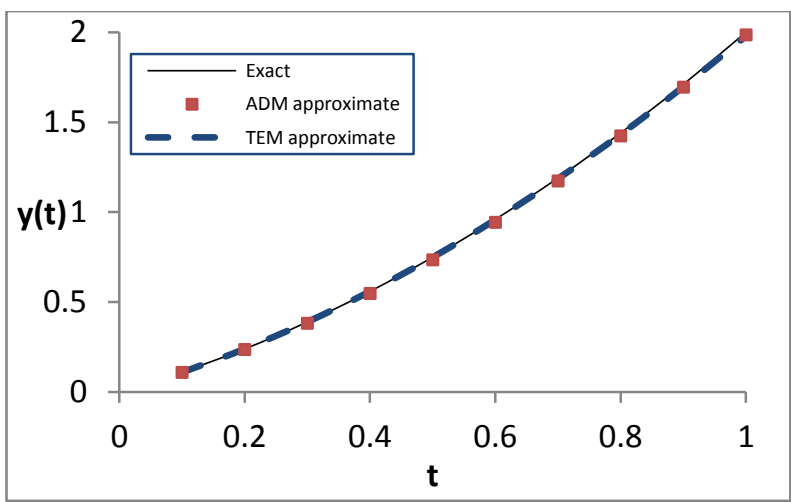

Fig.3. Comparison of approximate solutions obtained by ADM and TEM with exact solution of example 5.3.

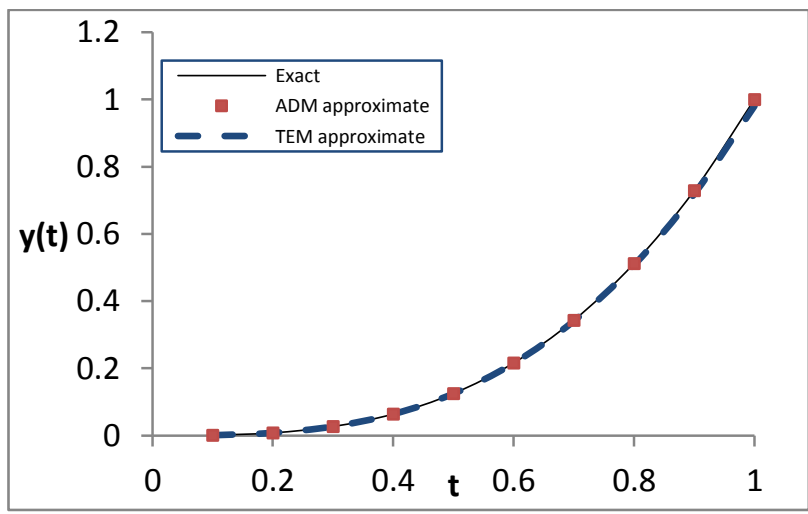

Fig.4. Comparison of approximate solutions obtained by ADM and TEM with exact solution of example 5.4.

\section{CONCLUSION}

In this paper, this study showed that for most problems the results in TEM are better than the results in ADM (see examples 1, 2, and 3). Except in the case of $y_{0}(t)$ equal to exact solution, find the results in ADM are the exact Solution (see example 4).

\section{ACKNOWLEDGMENTS}

Thanks to the experts who have contributed towards the development of the template.

\section{REFERENCES}

[1]A. Arikoglu, I. Ozkol, Solution of fractional integrodifferential equations by using fractional differential 
transform, Chaos, Solutions and Fractals, 40 (2009) 521529.

[2]Adam Loverro, Fractional calculus: History, Definitions and Application for the Engineer. (2004).

[3]A. Wazwaz, Areliable modification of Adomian decomposition method, Applied Mathematics and Computation, 102(1) (1999) 77-86.

[4]E. A. Rawashdeh, Numerical of fractional integrodifferential equations by collocation method, Appl. Math. Comput. 176 (2006) 1-6.

[5]E. A. Rawashdeh, Legendre Wavelet method for fractional integro-differential equations, Applied Mathematics Sciences.5 (2011) 2467-2474.

[6]F. Mainardi, Fractional calculus: Some basic problems in continuum and statistical mechanics, A carpinten and F. Mainardi (Eds), Fractals an Fractional Calulus in Continuum Mechanics, Spriger-verlag, New York, (1997) 291-348.

[7]I. Podlubny, Fractional Differential Equations, Academic press, New York, 1999.

[8]Jose' Paulo Carvalho dos Santos, M. Mallika Arjunan, Calaudio Cuevas, Existence results for fractional neutral integro-differential equations, Computers and Mathematics with Applications, 62 (2011) 1275-1283.

[9]K. Miller, B. Ross, An Introduction to the Fractional Calculus and Fractional Differential, Willey, New York, 1993.
[10]L. Huang, X. Li, Y. Zhao, X. Duan, Approximate solution of fractional integro-differential equations by Taylor expansion method, Computers and Mathematics with Application, 62 (2011) 1127-1134.

[11]M. Caputo, Linear models of dissipation whose $Q$ is almost frequecy independent-II, Geophysical Jornal of the Royal Astronomical Society, 13 (1967) 529-539.

[12]M. T. Rashed, Numerical solution of a special type of integro-differential equations, Appl. Math. Comput, 143 (2003) 73-88.

[13]R.C. Mittal, R. Nigam, Solution of fractional integrodifferential equations by Adomian decomposition method, Int. J. of Appl. Math. and Mech, 4 (2) (2008) 87 94.

[14]S. M. Momani, local and global existence theorems integro-differential equations, Jornal of Fractional Calculus, 18 (2000) 81-86.

[15]suayip Yüzbasl, Mehmer Sezer, Bayram Kemancl, Numerical solutions of integro-differential equations and application of apopulation model with an improved Legendere method, Applied Mathematics Modelling, 37 (2013) 2086-2101.

[16]W. E. Olmstead, R. A. Handelsman, Diffusion in a semiinfinite region with non linear dissipation, SIAM Rev. 18 (1976) 275-291. 